NISTIR 8016

\title{
A Proposed Interim Check for Field Testing a Laser Tracker's 3-D Length Measurement Capability Using a Calibrated Scale Bar as a Reference Artifact
}

\author{
Vincent D. Lee \\ Christopher Blackburn \\ Bala Muralikrishnan \\ Daniel Sawyer \\ Mark Meuret \\ Aaron Hudlemeyer
}


NISTIR 8016

\title{
A Proposed Interim Check for Field Testing a Laser Tracker's 3-D Length Measurement Capability Using a Calibrated Scale Bar as a Reference Artifact
}

\author{
Vincent D. Lee \\ Christopher Blackburn \\ Bala Muralikrishnan \\ Daniel Sawyer \\ Semiconductor and Dimensional Metrology Division \\ Physical Measurement Laboratory \\ Mark Meuret \\ Aaron Hudlemeyer \\ Brunson Instrument Company \\ Kansas City, $M O$
}

This publication is available free of charge from: http://dx.doi.org/10.6028/NIST.IR.8016

September 2014

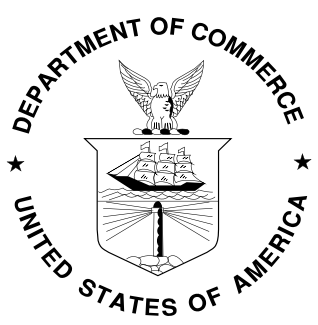

U.S. Department of Commerce

Penny Pritzker, Secretary

National Institute of Standards and Technology

Willie May, Acting Under Secretary of Commerce for Standards and Technology and Acting Director 


\title{
A Proposed Interim Check for Field Testing a Laser Tracker's 3-D Length Measurement Capability Using a Calibrated Scale Bar as a Reference Artifact
}

\author{
Vincent D. Lee, Christopher Blackburn, Bala Muralikrishnan, Daniel Sawyer \\ National Institute of Standards and Technology (NIST), Gaithersburg, MD, US \\ Mark Meuret, Aaron Hudlemeyer \\ Brunson Instrument Company, Kansas City, MO, US
}

\begin{abstract}
This paper describes a proposed interim check for field testing a laser tracker's 3-D length measurement capability using a calibrated scale bar as a reference artifact. The tests described here are constructed to be sensitive to uncompensated errors in length measurement caused by the imperfect geometry and optical misalignments in the laser tracker's construction. The collection of tests described in this paper was specifically selected to be sensitive to all of the error sources described in the laser tracker error models developed by the National Institute of Standards and Technology, and other researchers. Consequently, successful field test results obtained using the methods described here should provide operators with confidence that the instrument will perform within the required specification in the field. These tests are not a test of the tracker's ranging system, which is assumed to be individually calibrated and is used to establish metrological traceability per ASME B89.7.5 of the reference lengths described in this paper. To be of practical use to laser tracker users, the tests are constructed to be quick (less than 30 minutes) and easy to perform in the field while still providing a thorough evaluation of the instrument's length measurement performance. This paper describes an analytic method for selecting specific field tests, proposes a collection of field tests, and presents a method for displaying and analyzing the measurement data. ${ }^{1}$

\section{Introduction}

Performance testing of laser trackers is an important part of quality control for users of these systems. The results of these tests determine if an instrument is working within the required performance specifications. Since the introduction of laser trackers, numerous researchers have provided publications to help better understand the sources of measurement errors in these systems [1, 2]. Much of this information has been used to develop documentary standards (e.g. ASME B89.4.19, VDI/VDE 2617-10, ISO/DIS 10360-10) [3-5], which describe standard tests for evaluating instrument performance.
\end{abstract}

\footnotetext{
${ }^{1}$ Manufacturers may prescribe other methods for testing their trackers in the field; the tests described here are not designed to substitute for the manufacturer's more extensive evaluations or full parametric compensation.
} 
Tests in the published standards are comprised of a series of radial length measurements, transverse length measurements and two-face measurements. Radial length measurements test the interferometer (IFM) and/or absolute distance measurement (ADM) ranging system independently by measuring a calibrated length oriented collinear to the tracker's beam path. Transverse length measurements are performed by measuring calibrated lengths in orientations that are not parallel to the beam path. These orientations are also sensitive to the angle measuring systems in the tracker. The standards also include two-face tests, which involve measuring a stationary target, and then rotating the tracker's head $180^{\circ}$ in the horizontal plane and steering the laser beam (in the vertical plane) back toward the spherically mounted retroreflectors (SMR) [6]. The results from two-face testing are the distances between the two measured coordinates.

Both the ASME B89.4.19 and the ISO/DIS 10360-10 (currently a Draft International Standard) include a comprehensive set of performance tests. The full set of comprehensive tests outlined in the measurement standards require considerable time and effort to complete. Each document also includes a non-mandatory appendix describing an abbreviated test procedure called interim testing. While comprehensive testing per a measurement standard should be done occasionally, interim testing can be used as a quick check of the instrument in the field to determine if further testing is necessary. The ASME B89.4.19 interim tests provide a default set of measurement tests that are not fully sensitive to the error sources known to exist in laser tracker systems. While the interim test recommendations in the ISO/DIS 10360-10 include twoface tests and length tests, no specific guidance for choosing length tests is provided in the standard. This can be problematic because it is possible to select positions and orientations that are not sensitive to the major error sources in the specific laser tracker under test.

Well-constructed performance tests are sensitive to all the potential geometric misalignments in the measuring system under test. The tests in this document are designed to evaluate the tracker's general 3-D volumetric measurement capability. Consequently, radial measurements are not included. More precisely, the radial measurements, which are more accurate than the measurements that rely on the angular encoders, are performed to calibrate the reference length used to test the 3-D measurement performance of the laser tracker. It is assumed that the laser tracker's ranging system has been independently calibrated and has metrological traceability to the SI unit of length (the meter) through some other method; this is not uncommon.

\section{Selecting interim test measurements}

Geometric misalignments of the laser tracker's internal components exist due to the imperfect mechanical construction during assembly. NIST and others have developed models that describe how these misalignments contribute to measurement errors [7-9]. While the ASME 
and ISO laser tracker standards have suggestions for interim tests, no explicit information about the tests' sensitivity to the tracker misalignments is provided. The interim tests proposed in this paper are specifically chosen because of their sensitivity to all of the misalignments in the laser tracker models. Simulations were performed to determine a set of tests which optimize sensitivities to the geometric misalignments, while reducing the number of redundant measurements. The interim tests proposed in this paper are a collection of two-face and transverse length measurements.

Two-face tests are included in the proposed procedures because they are fast, easy to perform, and sensitive to many of the misalignments in the laser tracker. Because of the nature of these tests many of the measurement errors reverse in sign so the tests have high sensitivity to the misalignments.

Since two-face tests can be insensitive to certain misalignments in the device, transverse length measurements are also proposed. The targets are arranged vertically or horizontally so that ideally only one rotary axis of the machine is actuated at a time during measurement. Within these tests a subset of them will be symmetric length measurements, while another will be asymmetric. Symmetric length measurements place the tracker such that the targets are spaced equal distances away from tracker's center. Asymmetric measurements will place the device closer to one target relative to the other for a length measurement. Asymmetric length measurements have the appeal of being sensitive to larger number of misalignments than twoface or symmetric length measurements. Diagonal length measurements commonly found in the comprehensive performance test of the ASME and ISO standards are excluded because they are sensitive to the same errors that are captured by the other proposed tests.

For the interim tests proposed here, the tracker is placed $1 \mathrm{~m}$ from the scale bar for all tests. This eliminates the need to reposition the tracker, greatly reducing the time required to complete the test. A $1 \mathrm{~m}$ standoff distance allows for a large portion of the encoders to be tested, which increases sensitivity to certain misalignments.

The inclusion of additional tests would provide more information. However, one of the main goals is to establish a set of interim tests that allows for a quick but thorough evaluation of the tracker's performance in the field. Other tests can be selected using a similar approach if needed. The test described here are merely a recommendation that meet the requirements for sensitivity to the misalignments in the models employed.

Because the tests were designed to create procedures that are consistent from one test to the next, there are some redundant measurements. However, consistent procedures reduce the likelihood of measurement blunders-such as accidentally repeating or skipping over a required measurement. 


\section{Proposed tests and their sensitivities to laser tracker geometric misalignments}

The proposed interim test method utilizes a scale bar containing three kinematic seats that are arranged in a collinear fashion. Each kinematic seat holds an SMR such that the laser tracker can measure all three while stationary. The distance between the SMRs on the ends is nominally $2.3 \mathrm{~m}$ with a third SMR located approximately at the center of the bar (Figure 1).

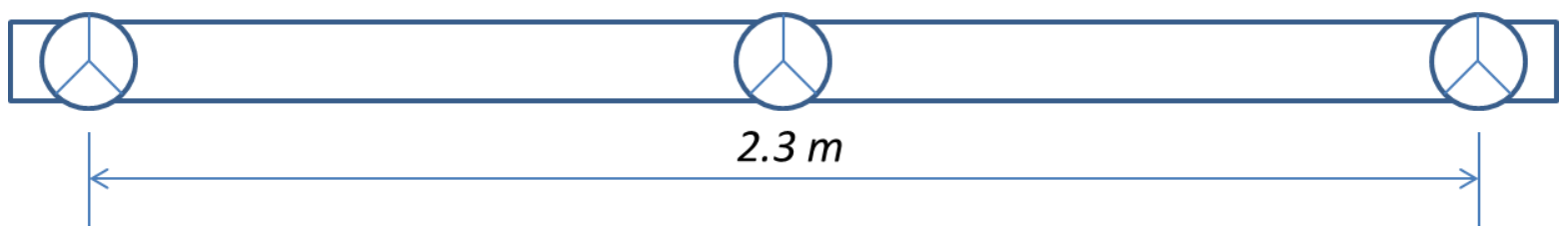

Figure 1: Scale bar with three kinematic seats holding SMRs

Calibration of this scale bar is typically performed using the ranging system of the laser tracker as described in section D-3 of ASME B89.4.19-2006. After calibration, the scale bar is measured in five different positions using the laser tracker's ADM system as described below and shown in Figure 2:

1. Scale bar in the vertical orientation, nest $\mathrm{B}$ at tracker height and the laser tracker at $0^{\circ}$ azimuth

2. Scale bar in the vertical orientation, nest $B$ at tracker height and the laser tracker at $90^{\circ}$ azimuth

3. Scale bar in the horizontal orientation, nest B at tracker height and the laser tracker at $90^{\circ}$ azimuth

4. Scale bar in the horizontal orientation, nest B at tracker height and the laser tracker at $0^{\circ}$ azimuth

5. Scale bar in the horizontal orientation, nest B at least $0.5 \mathrm{~m}$ above the laser tracker 


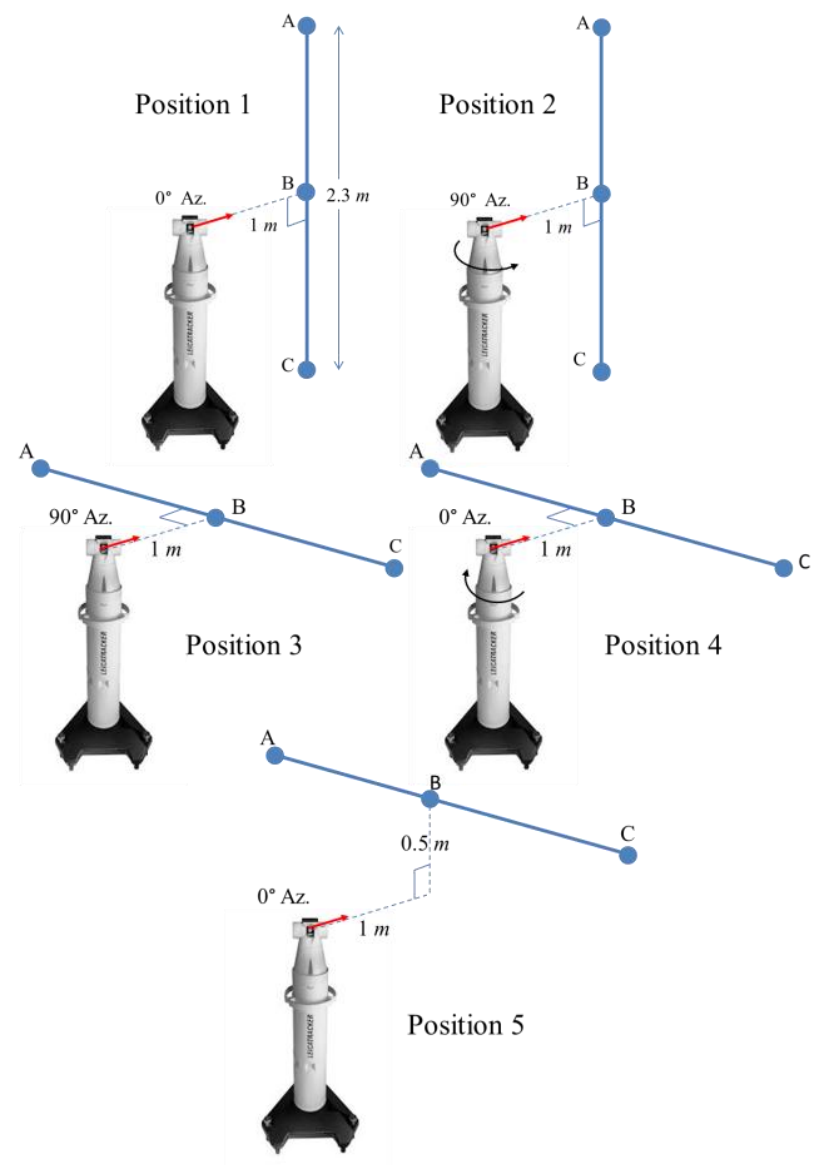

Figure 2: Five test positions to perform interim check of laser tracker

In each of these positions, the laser tracker measures each SMR with a two-face measurement. Once completed, 15 sets of two-face measurements, 5 symmetric length measurements and 10 asymmetric length measurements are calculated. Repeated measurements may also be performed. NIST performs three repeated measurements of the SMR in each position. This provides information on the repeatability of the instrument. A method for displaying and analyzing results is provided in the "Field Test Experiment" section below. The tests described above are performed using the laser tracker's ADM system. If the IFM will be used during actual measurements in the field, an additional test of the IFM zero setting should be performed ${ }^{2}$. To test for this error, the user should measure length AC (in IFM mode) with the scale bar in the horizontal position without breaking the measurement beam while moving the SMR from one end to the other.

Table 1 and Table 2 show the results of a sensitivity analysis for trackers without a beam steering mirror. The top row of variable names in these tables denotes each known geometric

\footnotetext{
2 The zero setting is often referred to as a "bird bath" error. This is the error in the determination of the distance from the tracker origin to the apex of the SMR when place in the home position.
} 
misalignment parameter in a laser tracker. The parameters are briefly described below with a more in-depth explanation by Muralikrishnan et al. [7]:

- $x 1 t$ and $x 1 m$ are beam offsets along transit axis and its normal

- $\mathrm{x} 2$ is the transit offset

- $x 5$ is the vertical index offset

- $x 6 t$ is the beam tilt (collimation error)

- $\quad x 8$ is the transit tilt (squareness)

- $x 9 x$ and $x 9 y$ are the horizontal angle encoder eccentricities along $x$ and $y$

- $x 10 n$ and $x 10 z$ are the vertical angle encoder eccentricities

- $x 11$ is the $R 0$ error

- $x 12 a$ and $x 12 b$ are the second order harmonic scale errors in the horizontal angle encoder scale

- $x 12 c$ and $x 12 d$ are the second order harmonic scale errors in the vertical angle encoder scale

The sensitivity numbers in these tables indicate how much a given misalignment will propagate into a measurement error for a specific position and test. Each sensitivity value denotes a certain error ratio. For example, in Table 2, the measurement of length $\mathrm{BC}$ with the scale bar in the horizontal elevated position has a sensitivity value of -0.7 for misalignment parameter $x 11$. This indicates that a $1 \mu \mathrm{m}$ misalignment of $\mathrm{x} 11$ produces a $-0.7 \mu \mathrm{m}$ error in the measured length. For misalignment parameters that describe angular misalignments, such as $x 2$, a sensitivity of -0.8 in the same measurement case means that a $1 \mu$ rad misalignment will translate to a $-0.8 \mu \mathrm{m}$ error in the measurement of length $B C$. All zeroes in any column means the set of tests have no sensitivity to the corresponding misalignments. (See the boxes in Table 1 and Table 3) 
Table 1: Two-face test sensitivity matrix for laser tracker without beam steering mirror

\begin{tabular}{|c|c|c|c|c|c|c|c|c|c|c|c|c|c|c|c|c|c|}
\hline & Targets & $\mathrm{Az}$ & $x 1 t$ & $\mathrm{x} 1 \mathrm{~m}$ & $x 2$ & $x 5$ & $x 6 t$ & $x 8$ & $x 9 x$ & x9y & $x 10 n$ & $x 10 z$ & $\mathrm{x} 11$ & x12a & $x 12 b$ & $\mathrm{x} 12 \mathrm{c}$ & $x 12 d$ \\
\hline Scale bar & A & 0 & 2 & 2 & 1.5 & 3 & 3 & 2.3 & 2 & 0 & 2.3 & 0 & 0 & 0 & 0 & 0 & 0 \\
\hline in vertical & B & 0 & 2 & 2 & 0 & 2 & 2 & 0 & 2 & 0 & 0 & 0 & 0 & 0 & 0 & 0 & 0 \\
\hline \multirow[t]{4}{*}{ orientation } & C & 0 & 2 & 2 & 1.5 & 3 & 3 & 2.3 & 2 & 0 & 2.3 & 0 & 0 & 0 & 0 & 0 & 0 \\
\hline & A & 90 & 2 & 2 & 1.5 & 3 & 3 & 2.3 & 0 & 2 & 2.3 & 0 & 0 & 0 & 0 & 0 & 0 \\
\hline & B & 90 & 2 & 2 & 0 & 2 & 2 & 0 & 0 & 2 & 0 & 0 & 0 & 0 & 0 & 0 & 0 \\
\hline & C & 90 & 2 & 2 & 1.5 & 3 & 3 & 2.3 & 0 & 2 & 2.3 & 0 & 0 & 0 & 0 & 0 & 0 \\
\hline Scale bar in & A & 90 & 2 & 2 & 0 & 3 & 3 & 0 & 2.3 & 2 & 0 & 0 & 0 & 0 & 0 & 0 & 0 \\
\hline horizontal & B & 90 & 2 & 2 & 0 & 2 & 2 & 0 & 0 & 2 & 0 & 0 & 0 & 0 & 0 & 0 & 0 \\
\hline \multirow[t]{4}{*}{ orientation } & C & 90 & 2 & 2 & 0 & 3 & 3 & 0 & 2.3 & 2 & 0 & 0 & 0 & 0 & 0 & 0 & 0 \\
\hline & A & 0 & 2 & 2 & 0 & 3 & 3 & 0 & 2 & 2.3 & 0 & 0 & 0 & 0 & 0 & 0 & 0 \\
\hline & B & 0 & 2 & 2 & 0 & 2 & 2 & 0 & 2 & 0 & 0 & 0 & 0 & 0 & 0 & 0 & 0 \\
\hline & C & 0 & 2 & 2 & 0 & 3 & 3 & 0 & 2 & 2.3 & 0 & 0 & 0 & 0 & 0 & 0 & 0 \\
\hline Scale bar in & A & 0 & 2 & 2 & 0.6 & 3.2 & 3.2 & 1 & 2 & 2.3 & 1 & 0 & 0 & 0 & 0 & 0 & 0 \\
\hline horizontal & B & 0 & 2 & 2 & 0.9 & 2.2 & 2.2 & 1 & 2 & 0 & 1 & 0 & 0 & 0 & 0 & 0 & 0 \\
\hline elevated & $\mathrm{C}$ & 0 & 2 & 2 & 0.6 & 3.2 & 3.2 & 1 & 2 & 2.3 & 1 & 0 & 0 & 0 & 0 & 0 & 0 \\
\hline
\end{tabular}

Table 2: Length test sensitivity matrix for laser tracker without beam steering mirror

Length Sensitivity matrix w/o beam steering mirror

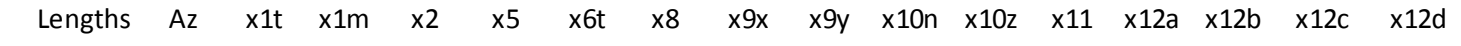

\begin{tabular}{|c|c|c|c|c|c|c|c|c|c|c|c|c|c|c|c|c|c|}
\hline Scale bar & $A C$ & 0 & 0 & 0 & 0 & 0 & 0 & 0 & 0 & 0 & 1.5 & 0 & -1.5 & 0 & 0 & 2 & 0 \\
\hline in vertical & $A B$ & 0 & 0 & 0.3 & 0 & 0 & 0 & 0 & 0 & 0 & 0.8 & 0.3 & -0.8 & 0 & 0 & 1 & 1.1 \\
\hline \multirow[t]{4}{*}{ orientation } & $B C$ & 0 & 0 & -0.3 & 0 & 0 & 0 & 0 & 0 & 0 & 0.8 & -0.3 & -0.8 & 0 & 0 & 1 & -1.1 \\
\hline & $A C$ & 90 & 0 & 0 & 0 & 0 & 0 & 0 & 0 & 0 & 1.5 & 0 & -1.5 & 0 & 0 & 2 & 0 \\
\hline & $A B$ & 90 & 0 & 0.3 & 0 & 0 & 0 & 0 & 0 & 0 & 0.8 & 0.3 & -0.8 & 0 & 0 & 1 & 1.1 \\
\hline & $B C$ & 90 & 0 & -0.3 & 0 & 0 & 0 & 0 & 0 & 0 & 0.8 & -0.3 & -0.8 & 0 & 0 & 1 & -1.1 \\
\hline Scale bar in & $A C$ & 90 & 0 & 0 & -1.5 & 0 & 0 & 0 & 1.5 & 0 & 0 & 0 & -1.5 & 2 & 0 & 0 & 0 \\
\hline horizontal & $A B$ & 90 & -0.3 & 0 & -0.8 & 0 & 0 & 0 & 0.8 & 0.3 & 0 & 0 & -0.8 & 1 & 1.1 & 0 & 0 \\
\hline \multirow[t]{4}{*}{ orientation } & $B C$ & 90 & 0.3 & 0 & -0.8 & 0 & 0 & 0 & 0.8 & -0.3 & 0 & 0 & -0.8 & 1 & -1.1 & 0 & 0 \\
\hline & $A C$ & 0 & 0 & 0 & -1.5 & 0 & 0 & 0 & 0 & 1.5 & 0 & 0 & -1.5 & -2 & 0 & 0 & 0 \\
\hline & $A B$ & 0 & -0.3 & 0 & -0.8 & 0 & 0 & 0 & -0.3 & 0.8 & 0 & 0 & -0.8 & -1 & -1.1 & 0 & 0 \\
\hline & $B C$ & 0 & 0.3 & 0 & -0.8 & 0 & 0 & 0 & 0.3 & 0.8 & 0 & 0 & -0.8 & -1 & 1.1 & 0 & 0 \\
\hline Scale bar in & $A C$ & 0 & 0 & 0.5 & -1.5 & -0.8 & 0 & 0 & 0 & 1.5 & -0.2 & 0.7 & -1.4 & -2 & 0 & -0.4 & 0.6 \\
\hline horizontal & $A B$ & 0 & -0.3 & 0.2 & -0.8 & -0.4 & -0.1 & -0.2 & -0.3 & 0.8 & -0.1 & 0.4 & -0.7 & -1 & -1.1 & -0.2 & 0.3 \\
\hline elevated & $\mathrm{BC}$ & 0 & 0.3 & 0.2 & -0.8 & -0.4 & 0.1 & 0.2 & 0.3 & 0.8 & -0.1 & 0.4 & -0.7 & -1 & 1.1 & -0.2 & 0.3 \\
\hline
\end{tabular}

A similar sensitivity analysis was performed for laser trackers with a beam steering mirror mechanism; those results are shown below in Table 3 and Table 4. Each geometric error parameter for this type of laser tracker is briefly described below and further details can be found in a publication by Loser and Kyle [8]:

- $\mathrm{x} 1 \mathrm{x}$ and $\mathrm{x} 1 \mathrm{y}$ are beam offsets along $\mathrm{x}$ and $\mathrm{y}$ relative to the mirror's center of rotation

- $\quad \mathrm{x} 2$ is the transit offset

- $x 3$ is the mirror offset 
- $\quad x 4 t$ and $x 4 n$ are the cover plate offsets (caused by refraction of the beam exiting body of laser tracker through an aperture)

- $x 5$ is the vertical index offset

- $\quad x 6 x$ and $x 6 y$ are the beam tilt terms

- $\quad x 7$ is the mirror tilt (collimation error)

- $\quad x 8$ is the transit tilt (squareness)

- $\quad x 9 x$ and $x 9 y$ are the horizontal angle encoder eccentricities along $x$ and $y$

- $x 10 n$ and $x 10 z$ are the vertical angle encoder eccentricities

- $x 11$ is the $R 0$ error

- $x 12 a$ and $x 12 b$ are the second order harmonic scale errors in the horizontal angle encoder scale

- $\quad x 12 c$ and $x 12 d$ are the second order harmonic scale errors in the vertical angle encoder scale

Table 3: Two-face test sensitivity matrix for LT with beam steering mirror

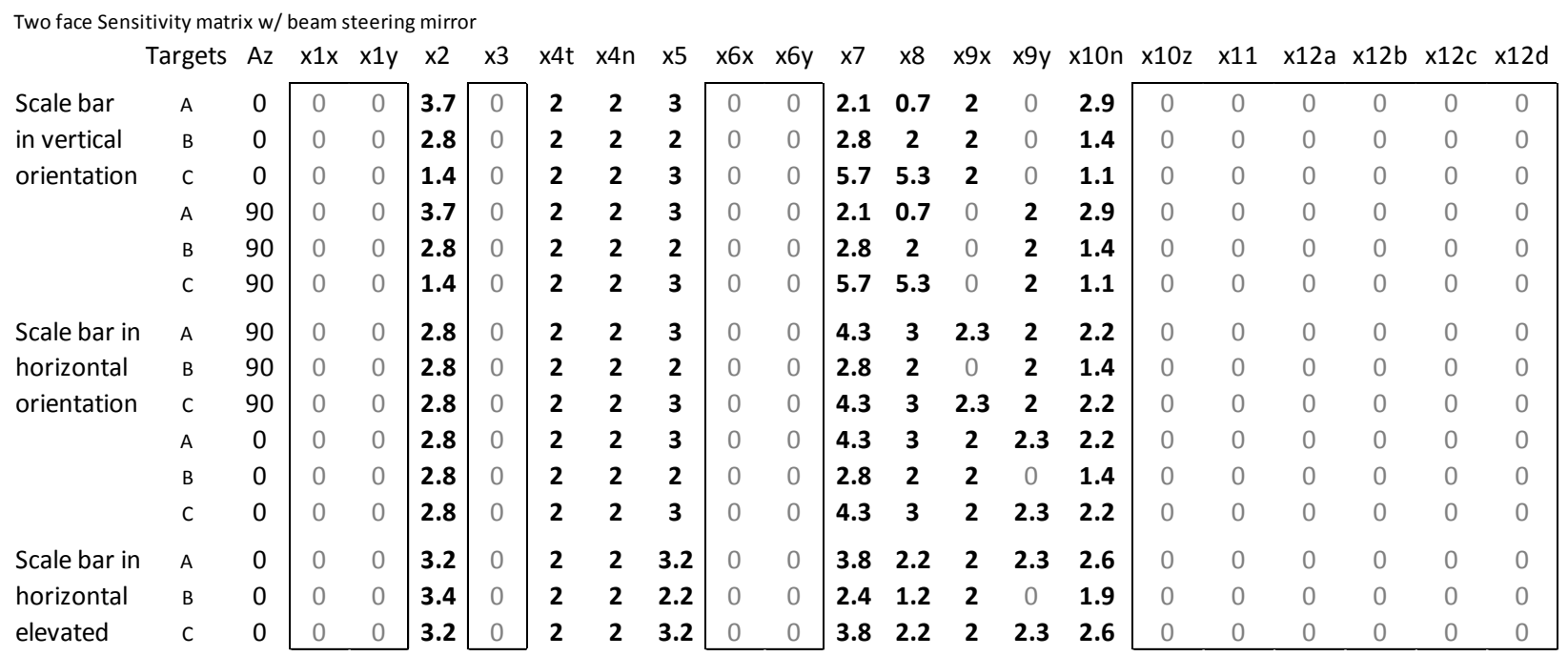


Table 4: Length test sensitivity matrix for LT with beam steering mirror

\begin{tabular}{|c|c|c|c|c|c|c|c|c|c|c|c|c|c|c|c|c|c|c|c|c|c|c|}
\hline & Lengths & $\mathrm{Az}$ & $x 1 x$ & $x 1 y$ & $x 2$ & x3 & $x 4 t$ & $x 4 n$ & x5 & $x 6 x$ & $x 6 y$ & $x 7$ & x8 & $x 9 x$ & $x 9 y$ & $\mathrm{x} 10 \mathrm{n}$ & $x 10 z$ & x11 & x12a & $x 12 b$ & $\mathrm{x} 12 \mathrm{c}$ & $x 12 d$ \\
\hline \multirow{6}{*}{$\begin{array}{l}\text { Scale bar } \\
\text { in vertical } \\
\text { orientation }\end{array}$} & $A C$ & 0 & 0 & 0 & 0 & -1.2 & 0 & 0 & 0 & 0 & 0 & 0 & 0 & 0 & 0 & 0.6 & 0.6 & -1.5 & 0 & 0 & 0 & 1.5 \\
\hline & $A B$ & 0 & 0 & 0.3 & -0.3 & -0.7 & 0 & 0.3 & 0 & 0 & 0 & 0 & 0 & 0 & 0 & 0.2 & 0.4 & -0.8 & 0 & 0 & -0.3 & 0.8 \\
\hline & $B C$ & 0 & 0 & -0.3 & 0.3 & -0.5 & 0 & -0.3 & 0 & 0 & 0 & 0 & 0 & 0 & 0 & 0.4 & 0.2 & -0.8 & 0 & 0 & 0.3 & 0.8 \\
\hline & $A C$ & 90 & 0 & 0 & 0 & -1.2 & 0 & 0 & 0 & 0 & 0 & 0 & 0 & 0 & 0 & 0.6 & 0.6 & -1.5 & 0 & 0 & 0 & 1.5 \\
\hline & $A B$ & 90 & 0.3 & 0 & -0.3 & -0.7 & 0 & 0.3 & 0 & 0 & 0 & 0 & 0 & 0 & 0 & 0.2 & 0.4 & -0.8 & 0 & 0 & -0.3 & 0.8 \\
\hline & $\mathrm{BC}$ & 90 & -0.3 & 0 & 0.3 & -0.5 & 0 & -0.3 & 0 & 0 & 0 & 0 & 0 & 0 & 0 & 0.4 & 0.2 & -0.8 & 0 & 0 & 0.3 & 0.8 \\
\hline \multirow{6}{*}{$\begin{array}{l}\text { Scale bar in } \\
\text { horizontal } \\
\text { orientation }\end{array}$} & $A C$ & 90 & 1 & 0 & -1.5 & $\mid-2.1$ & 0 & 0 & 0 & -1.5 & 0 & 0 & 0 & 1.5 & 0 & 0 & 0 & -1.5 & 2 & 0 & 0 & 0 \\
\hline & $A B$ & 90 & 0.5 & 0.6 & -0.8 & -1.1 & 0.3 & 0 & 0 & -0.8 & -0.3 & 0 & 0 & 0.8 & 0.3 & 0 & 0 & -0.8 & 1 & 1.1 & 0 & 0 \\
\hline & $B C$ & 90 & 0.5 & -0.6 & -0.8 & -1.1 & -0.3 & 0 & 0 & -0.8 & 0.3 & 0 & 0 & 0.8 & -0.3 & 0 & 0 & -0.8 & 1 & -1.1 & 0 & 0 \\
\hline & $A C$ & 0 & 0 & 1 & -1.5 & -2.1 & 0 & 0 & 0 & 0 & -1.5 & 0 & 0 & 0 & 1.5 & 0 & 0 & -1.5 & -2 & 0 & 0 & 0 \\
\hline & $A B$ & 0 & -0.6 & 0.5 & -0.8 & -1.1 & 0.3 & 0 & 0 & 0.3 & -0.8 & 0 & 0 & -0.3 & 0.8 & 0 & 0 & -0.8 & -1 & -1.1 & 0 & 0 \\
\hline & $B C$ & 0 & 0.6 & 0.5 & -0.8 & -1.1 & -0.3 & 0 & 0 & -0.3 & -0.8 & 0 & 0 & 0.3 & 0.8 & 0 & 0 & -0.8 & -1 & 1.1 & 0 & 0 \\
\hline \multirow{3}{*}{$\begin{array}{l}\text { Scale bar in } \\
\text { horizontal } \\
\text { elevated }\end{array}$} & $A C$ & 0 & 0 & 1.3 & -2 & -2.4 & 0 & 0.5 & -0.8 & 0 & -2.1 & 0 & 0 & 0 & 1.5 & -0.6 & 0.4 & -1.4 & -2 & . & -0.7 & -0.2 \\
\hline & $A B$ & 0 & -0.7 & 0.6 & -1 & -1.2 & 0.3 & 0.2 & -0.4 & 0.7 & -1 & 0.1 & 0.1 & -0.3 & 0.8 & -0.3 & 0.2 & -0.7 & -1 & -1.1 & -0.4 & -0.1 \\
\hline & $B C$ & 0 & 0.7 & 0.6 & -1 & -1.2 & -0.3 & 0.2 & -0.4 & -0.7 & -1 & -0.1 & -0.1 & 0.3 & 0.8 & -0.3 & 0.2 & -0.7 & -1 & 1.1 & -0.4 & -0.1 \\
\hline
\end{tabular}

The preceding sensitivity analysis was performed for a laser tracker measuring the artifact shown in Figure 1 and for the positions shown in Figure 2 and is only valid for this case. Manufacturers typically specify two-face tests due to their sensitivity to a large number of errors. However, observations of the data presented in Table 1 and Table 3 above, show that two-face tests are not sensitive to the second order harmonics in the horizontal and vertical angle encoders (highlighted by the boxed in area). Additionally, in the case of a laser tracker with a beam steering mirror, two-face tests are not sensitive to beam offsets and beam tilt errors ( $x 1 x, x 1 y, x 6 x$, and $x 6 y)$ as shown in Table 4. The addition of length measurements captures the effects of geometrical misalignments that do not manifest themselves in two-face tests (boxed in portions of Table 2 and Table 4). The collection of both of these tests is sensitive to all the misalignments described in [7-9] and comprises of a thorough evaluation of a laser tracker's performance.

\section{Field Test Experiment}

This section provides a sample of results obtained from performing the proposed interim tests. These tests were performed at NIST in a controlled environment (humidity controlled to $50 \%$ $\mathrm{RH}$, and temperature to $\left.(20 \pm 0.5)^{\circ} \mathrm{C}\right)$, and represent the tracker's performance under ideal measurement conditions. The results presented may not be achievable under significantly different environmental conditions. The measurements were taken using a single laser tracker without a beam steering mirror. This data was collected over the course of two weeks and shows the variation in the device's performance over this time interval. The data is presented graphically for ease of interpretation. Two separate charts are provided simply for ease of readability. A chart for the two-face test results and a chart for the length test results are 
provided below. Each line on the charts represents one full run of the interim tests on the day specified and each data point represents the largest error of three repeats.

Figure 3 shows the results of the two-face tests. Because the results of these tests are distances, the errors are always positive. It is interesting to note that with the scale bar mounted vertically there are large and repeatable errors with the SMR measured in the lowest nest (nest $C$ in Figure 2). Table 2 shows that this test is sensitive to a number of misalignments so the source of these large errors cannot be determined. For the testing described in this document it is only important that the tests are sensitive to all the misalignmentscompensation using other methods may be required if the errors are unacceptably large.

Figure 4 shows the results of the length tests. Unlike the two-face results, these test results may be positive or negative. The chart shows measurement results that are somewhat biased in the positive. Table 3 shows that the length measurements are sensitive to $x 11$, which is the R0 or birdbath error. Furthermore, the sensitivities have the same sign for all of the tests. This implies that, if present, this error source could be responsible for some of the bias in the data. Further testing is needed to determine if $x 11$ is the source of the bias.

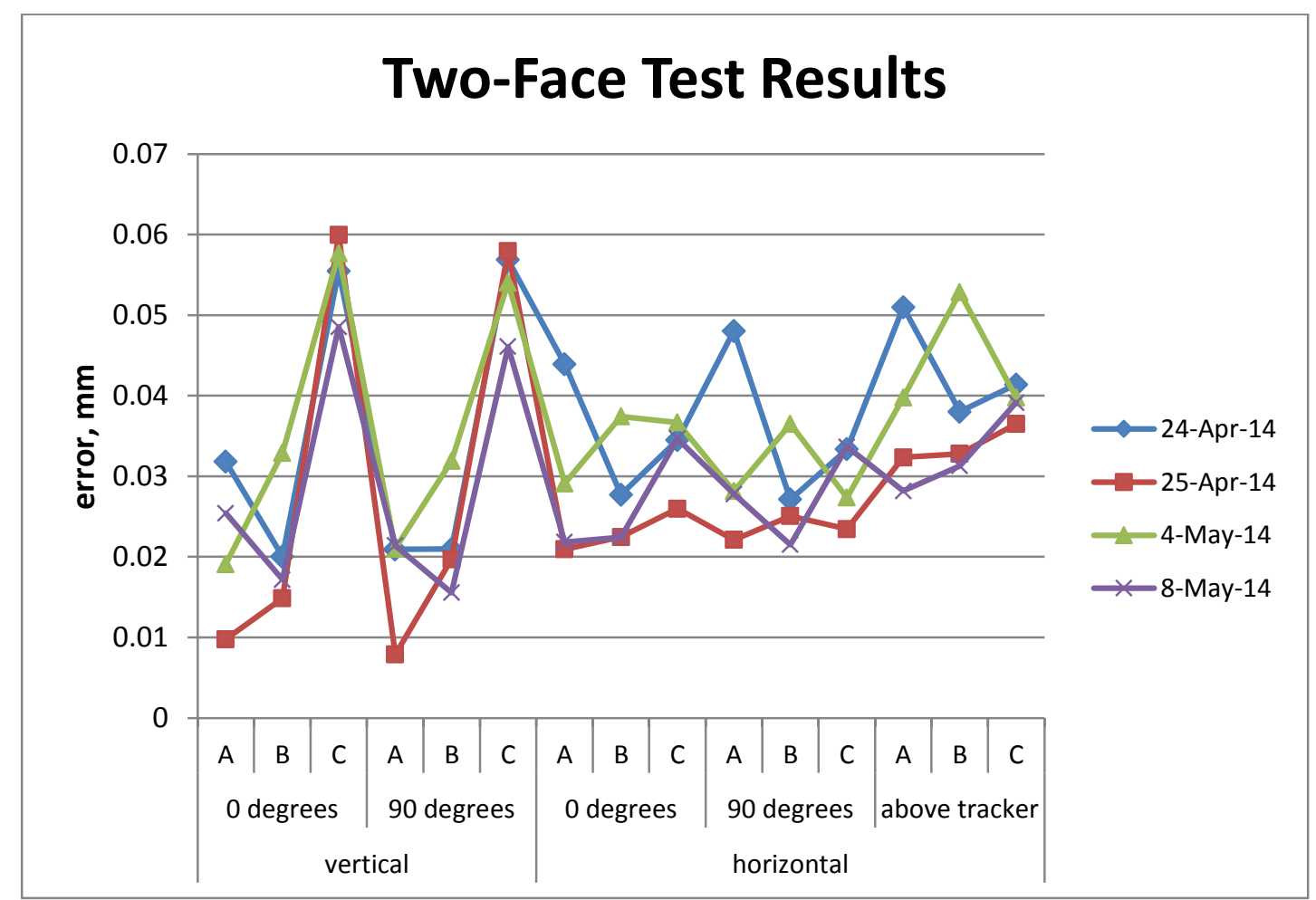

Figure 3: Test results for two-face measurements 


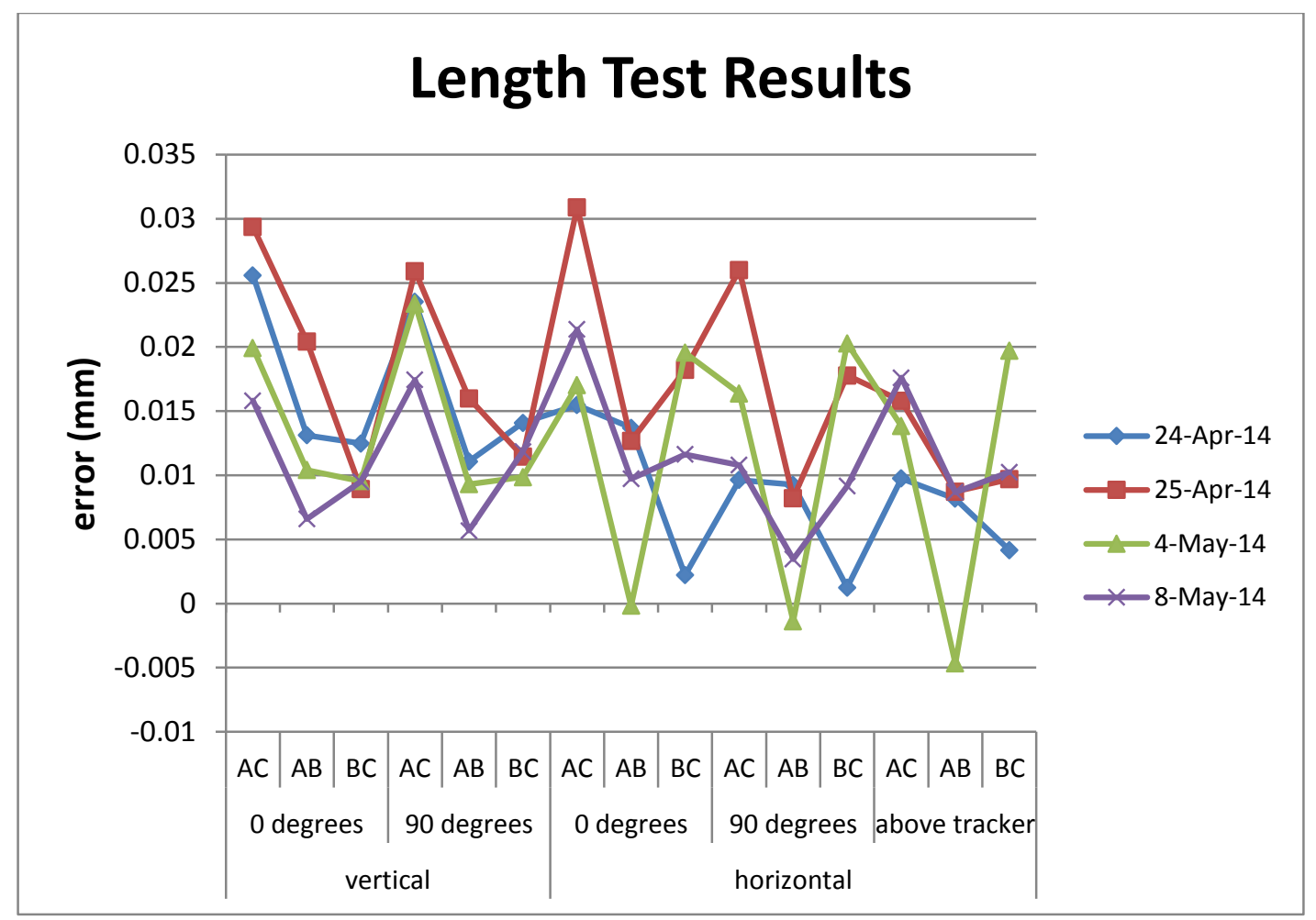

Figure 4: Test results for length measurements

By periodically performing this test and plotting the data, the instrument user can monitor the instrument's performance over time. As more data is collected, trends can be established to denote the overall health of the laser tracker. Should the device start to deviate from the user's performance requirements corrective actions can be taken. This data can also be used to determine the required calibration intervals, which may be very different for different measurement tasks.

\section{Conclusion}

The interim check described in this paper provides a method for users of laser trackers to quickly check that their device is performing within required specifications. The various measurements in the proposed interim tests were chosen based on simulations using NIST's and established laser tracker models, and are designed to expose internal mechanical misalignments in the form of length and position measurement errors. These tests measure a scale bar in five different orientations relative to the laser tracker. The battery of tests involves a series of length measurements and two-face tests. A commercially available laser tracker system was tested with the interim test procedure, and a method for collecting and displaying the data was provided. The proposed interim tests can typically be completed in 30 minutes or less by an experienced user. 


\section{References}

[1] H. Zhuang and Z. Roth, "Modeling Gimbal Axis Misalignments and Mirror Center Offset in a Single-beam Laser Tracking Measurement System," International Journal of Robotics, vol. 14, pp. 211-224, 1995.

[2] B. Hughes, A. Forbes, A. Lewis, W. Sun, D. Veal, and K. Nasr, "Laser traker error determination using a network mesurement," Measurement Science and Technology, vol. 22, 2011.

[3] ASME, "B89.4.19-2006: Performance Evaluation of Laser-Based Spherical Coordinate Measurment Systems," ed. New York, USA: American Society of Mechanical Engineers, 2006.

[4] VDI/VDE, "VDI/VDE 2617 Part 10: Accuracy of Coordinate Measuring Machines: Characteristics and their Checking: Acceptance and Reverification Tests of Laser Trackers," ed. Dusseldorf: ISO, 2001.

[5] ISO, "ISO/DIS 10360-10: Geometrical product specifications (GPS)-acceptance and reverification tests for coordinate measuring systems (CMS): part 10. Laser trackers for measuring point-to-point distances," ed. Geneva, Switzerland: International Standards Orginization, 2013.

[6] S. K. Duggal, Surveying, 2nd ed. New Delhi India: Tata McGraw-Hill, 2004.

[7] B. Muralikrishnan, D. Sawyer, C. Blackburn, S. Phillips, B. Borchardt, and W. T. Estler, "ASME B89.4.19 Performance Evaluation Tests and Geometric Misalignments in Laser Trackers," Journal of Research of the National Institute of Standards and Technology, vol. 114, pp. 21-35, 2009.

[8] R. Loser and S. Kyle, "Alignment and field check procedures for the Leica Laser Tracker LTD 500," in Boeing Large Scale Optical Metrology Seminar, 1999.

[9] B. Muralikrishnan, D. Sawyer, C. Blackburn, S. Phillips, R. Bridges, and M. Quan, "Measuring Scale Errors in a Laser Tracker's Horizontal Angle Encoder Through Simple Length Measurement and Two-Face System Tests," Journal of Research of the National Institute of Standards and Technology, vol. 115, pp. 291-301, 2010. 